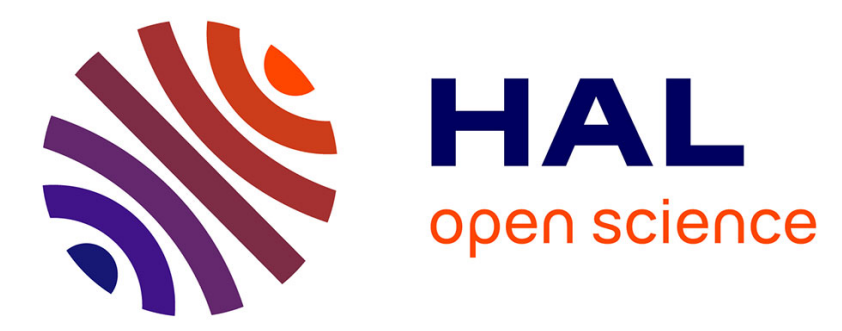

\title{
La stabilisation des prix aux producteurs agricoles: approches micro-économiques
}

\author{
Catherine Araujo Bonjean, Jean-Marc Boussard
}

\section{To cite this version:}

Catherine Araujo Bonjean, Jean-Marc Boussard. La stabilisation des prix aux producteurs agricoles: approches micro-économiques. Revue Tiers Monde, 1999, 40 (160), pp.901-928. 10.3406/tiers.1999.5351. hal-02688099

\section{HAL Id: hal-02688099 \\ https://hal.inrae.fr/hal-02688099}

Submitted on 1 Jun 2020

HAL is a multi-disciplinary open access archive for the deposit and dissemination of scientific research documents, whether they are published or not. The documents may come from teaching and research institutions in France or abroad, or from public or private research centers.
L'archive ouverte pluridisciplinaire HAL, est destinée au dépôt et à la diffusion de documents scientifiques de niveau recherche, publiés ou non, émanant des établissements d'enseignement et de recherche français ou étrangers, des laboratoires publics ou privés.

\section{(1) (1) $\$$}

Distributed under a Creative Commons Attribution - NonCommercial - NoDerivatives $\mid 4.0$ 


\section{La stabilisation des prix aux producteurs agricoles : approches} micro-économiques

Catherine Araujo Bonjean, Jean-Marc Boussard

\section{Citer ce document / Cite this document :}

Araujo Bonjean Catherine, Boussard Jean-Marc. La stabilisation des prix aux producteurs agricoles : approches microéconomiques. In: Tiers-Monde, tome 40, n¹60, 1999. Études sur la pauvreté, prix agricoles et filières intégrées, nationalistes hindous et développement... pp. 901-928;

doi : https://doi.org/10.3406/tiers.1999.5351

https://www.persee.fr/doc/tiers_1293-8882_1999_num_40_160_5351

Fichier pdf généré le 30/03/2018 


\title{
LA STABILISATION DES PRIX AUX PRODUCTEURS AGRICOLES : APPROCHES MICRO-ÉCONOMIQUES
}

\author{
par Catherine Araujo Bonjean, Jean-Marc Boussard*
}

Depuis le début des années 80, en Afrique, de nombreuses institutions qui avaient été mises en place dans les années 50 et 60 pour stabiliser les revenus des producteurs agricoles ont été démantelées en raison de leur inefficacité. On peut cependant se demander si ces suppressions, pour justifiées qu'elles aient été, n'ont pas créé un vide à combler, dès lors $q u$ 'il existe des raisons profondes pour la stabilisation. Une revue de la littérature sur le sujet montre en effet que le rôle joué par un organisme de stabilisation peut être hénéfique sous certaines conditions, en particulier lorsque les pays sont soumis à des risques non assurables.

Face aux conséquences supposées ou avérées néfastes de l'instabilité des revenus agricoles aux niveaux macro- et microéconomiques, la majorité des pays d'Afrique au sud du Sahara ont mis en place dans les années 50 des mécanismes publics de gestion de l'instabilité des prix et des productions agricoles. Ces mécanismes, stocks régulateurs pour les produits vivriers échangés au niveau national, caisses de stabilisation ou de péréquation pour les produits échangés sur les marchés internationaux, ont fait l'objet de profondes réformes au cours des années 80, conduisant souvent à leur suppression. Pourtant, l'échec de ces organismes apparaît avoir été davantage la conséquence de leur mauvaise gestion, et leur réforme plus dictée par un souci d'économie budgétaire que par la disparition des causes profondes qui avaient motivé leur création.

Il est donc utile de revenir sur le problème de l'instabilité agricole, d'abord pour savoir si elle est véritablement pernicieuse (après tout,

* Respectivement, (NRS-(CRIDI. Clermont-Ferrand. et INRA-STEPF. Paris.

Rowe Ticrs Monde. t. XI.. n" 160. septembre-décembre 1999 
peut-être a-t-elle de bons côtés ?), ensuite, si elle est aussi mauvaise qu'on le dit, pour voir comment la supprimer, ou tout au moins la réduire. Aussi bien, pour réduire l'instabilité, faut-il d'abord en comprendre l'origine. Ce document sera organisé en trois parties, la première consacrée aux effets de l'instabilité, la seconde à ses causes, la troisième aux moyens de l'atténuer.

\section{LES EFFETS DE L'INSTABILITÉ}

Cette question a été passionnément discutée depuis la grande crise de 1929 et l'intervention massive des États dans l'agriculture qui s'en est suivie dans tous les pays développés. Les résultats varient beaucoup suivant le cadre d'hypothèse choisi. Nous distinguons ici les effets de l'instabilité sur le bien-être des producteurs et ses effets sur l'offre agricole.

\section{Le coût du risque pour les producteurs agricoles}

Après les travaux pionniers de Waugh (1944), Oi (1961) et Massell (1969), fondés sur l'analyse marshallienne des surplus, le cadre général de référence de l'analyse coût/bénéfice de la stabilisation des prix est celui développé par Newbery et Stiglitz en 1981. C'est une analyse statique qui ne prend pas en compte la réponse de l'offre à la stabilisation des prix et dans laquelle les producteurs et les consommateurs sont considérés comme deux entités isolées. Pour définir le coût du risque, et par conséquent le bénéfice de stabilisation, il est nécessaire d'introduire d'abord une digression sur la notion de risque et d'aversion au risque.

\section{La prime de risque}

Le coût du risque, ou prime de risque, est donné par la perte de revenu moyen qu'un individu est disposé à consentir pour échanger un revenu aléatoire contre un revenu certain. De fait, pour le producteur qui a de l'aversion à l'égard du risque, il n'est pas équivalent d'avoir $500 \mathrm{~F}$ à coup sûr ou une chance sur deux d'avoir $1000 \mathrm{~F}$. L'équivalent certain de la loterie qui donne une chance sur deux d'avoir $1000 \mathrm{~F}$ n'est pas $500 \mathrm{~F}$ (l'espérance mathématique) mais une somme inférieure, qui dépend de l' "aversion pour le risque" du décideur. Par exemple $400 \mathrm{~F}$, si le décideur a peu d'aversion pour le risque, ou $200 \mathrm{~F}$ s'il en a beaucoup. Ainsi, le décideur dont l'équivalent certain, dans 
l'exemple précédent, est de $400 \mathrm{~F}$, est-il disposé à sacrifier $100 \mathrm{~F}$ d'espérance de gain contre la certitude de gagner à la loterie. Sa prime de risque, définie comme l'écart entre le revenu certain et l'espérance du revenu aléatoire, est ici égale à $100 \mathrm{~F}$.

Cette prime de risque dépend de l'importance du risque auquel est soumis le producteur et de son aversion au risque. Il est important de noter à ce sujet que l'aversion pour le risque dépend de la richesse du décideur. Un agriculteur endetté, dont la richesse est négative, n'aura pas le même comportement qu'un agriculteur dont le compte en banque est bien garni'. On verra plus loin les conséquences pratiques de ce phénomène.

Il existe de nombreuses mesures du risque ${ }^{2}$, et de nombreuses controverses entre les économistes sur «le comportement de l'homme rationnel devant le risque ". On trouvera plus de détails sur la mesure du risque chez Rotschild et Stiglitz (1970), Newbery et Stiglitz (1981), et des études de cas sur la mesure du risque chez Newbery et Stiglitz (1981), Badillo et Dalloz (1985), Roumasset et al. (1979), etc. Parmi les études empiriques sur l'aversion au risque des producteurs agricoles on retiendra les études de cas suivantes : l'étude de Dillon et Scandizzo (1978) sur le Brésil, l'étude de Binswanger (1980, 1981) sur l'Inde, l'étude de Moscardi et de Janvry (1977) sur le Mexique, l'étude de Boussard et Petit (1967) sur la France. Ces études font généralement apparaître de l'aversion pour le risque.

\section{Le bénéfice de la stabilisation des prix}

Le bénéfice de la stabilisation des prix pour le producteur est égal à la somme qu'il serait prêt à payer pour que soit introduit un mécanisme de stabilisation. Il se compose d'un "bénéfice de transfert " (variation du revenu moyen du producteur engendrée par le mécanisme de stabilisation et compensée par une variation équivalente de la dépense des consommateurs) et d'un "bénéfice d'efficience", ou prime de risque, qui résulte de la réduction d'un risque coûteux pour l'individu?. Il est

1. La prime de risque est définie par : $:=\frac{1}{2} A$ var (w) où var (w) est la variance de la richesse et $A$ le coefficient daversion absolue pour le risque : $\mathrm{A}=\frac{U^{\prime \prime \prime}(W)}{U^{\prime \prime}(W)}$, ou $U(W)$ est lutilité de la richesse $W$ du décideur. A est généralement considéré comme décroissant avec le niveau de richesse de l'agent (Laffont, $1985)$.

2. Cf.. par ex. Munier. 1988, ou Roumasset, Boussard, Singh, 1979, ou encore, le livre récent de Hardaker et al. 1997.

3. Le bénéfice de stabilisation est donné par: $B=د P-\frac{1}{2} R \Delta \delta ; P$, avec $P$ : revenu moyen, $R$ : coefficient daversion relative au risque, $x_{i}^{2}$ : coefficient de variation du revenu. 
important de rappeler que cette mesure du bénéfice de stabilisation ignore la réponse de l'offre à la stabilisation des prix.

Les résultats des évaluations faites par Newbery et Stiglitz au niveau international de la stabilisation des prix de 6 produits de base et pour 14 PVD tendent à montrer que les bénéfices de stabilisation sont faibles par rapport au coût de fonctionnement d'un mécanisme régulateur et qu'ils ne profitent pas nécessairement aux producteurs. À moins que ces derniers n'aient une très forte aversion pour le risque, le bénéfice net de la stabilisation des prix est négatif pour les producteurs. Dans tous les cas, les consommateurs sont les principaux gagnants d'une stabilisation des prix.

En particulier, le bénéfice net de la stabilisation des prix peut être très grand pour des produits vivriers, notamment si l'on prend en compte le point de vue des pauvres', et si l'on tient compte du fait qu'il existe d'autres méthodes de stabilisation que le stockage/déstockage, comme, par exemple, les quotas de production, discutés ci-après. Pour les consommateurs de produits vivriers, les pertes liées au risque de prix sont toujours plus importantes que les gains du producteur et sont spécialement sévères pour les consommateurs pauvres. Pour ceuxci, une hausse de prix représente une amputation d'autant plus forte de leur revenu que la part de l'alimentation dans ce revenu est plus élevé. Le résultat peut parfois conduire à une augmentation de la consommation de certaines denrées alimentaires lorsque les prix montent (effet Giffen), ce qui contribue à accroître la crise au lieu de la résorber ${ }^{2}$.

De même, si l'on considère le ménage paysan en tant qu'unité de production et de consommation, la prime de risque tend à être plus élevée pour le ménage que pour un producteur pur lorsque le prix des productions vivrières est risqué ${ }^{3}$. Autrement dit, l'effet négatif du risque sur le bien-être du ménage est plus grand que pour le producteur.

Parmi les évaluations empiriques du bénéfice de stabilisation, on citera les travaux de Braverman et al. (1990) pour le Brésil, et de Bonjean (1994) pour la Côte-d'Ivoire et Madagascar. Partant des analyses de Newbery et Stiglitz, les auteurs ont étudié les conséquences de la stabilisation des prix de différentes cultures au niveau du pays produc-

1. Newbery, $1989 a$.

2. Sir Robert Giffen, en étudiant le comportement des classes pauvres en Angleterre au début du $X X^{\circ}$ siècle, avait montré que la hausse du prix du pain. en diminuant leurs revenus réels, obligeait ces consommateurs à renoncer au pauvre luxe qui consistait à se payer un peu de viande de temps à autre. Mais cette réduction de la consommation de viande, elle-même, les entrainait à accroitre leur consommation de pain, pour maintenir la quantité totale de calories ingérée.

3. Finkelshtain et Chalfant, 1991 
teur. Pour Madagascar et la Côte-d'Ivoire, bien qu'une politique de stabilisation totale ou partielle des prix des produits d'exportation (indexés sur un prix de référence défini comme une moyenne mobile des prix passés) apparaisse désirable pour les producteurs, les déficits importants qu'elle entraîne rendent une telle politique peu réaliste.

\section{Conséquences de l'instabilité sur l'offre}

La plupart des études sur cette question ont considéré l'incidence de l'instabilité du revenu sur l'offre du produit risqué dans le cadre d'un modèle de producteur " pur ». Cependant, en cas de défaillances de marchés, il peut être impossible de considérer séparément les décisions de production et de consommation. En particulier, l'absence de marché du crédit, ou les coûts élevés de transaction sur ce marché, brisent la récursivité du modèle de ménage!.

a) La réponse des producteurs face au risque de revenu

L'évaluation de la réponse des producteurs face à l'instabilité de leurs revenus dépend des hypothèses faites sur leur attitude face au risque et sur l'ouverture du marché vis-à-vis de l'extérieur². On distingue habituellement quatre cas de figure selon que le producteur a ou non de l'aversion pour le risque et selon que les prix et les quantités sont ou non négativement corrélés ${ }^{3}$.

\section{Hypothèse de neutralité du producteur face au risque}

Dans l'hypothèse où les producteurs sont " neutres " vis-à-vis du risque, ils sont prêts à jouer à n'importe quel jeu de hasard pourvu que l'espérance de gain soit supérieure à la mise. La variabilité du gain

1. In modèle de ménage est dit séparable ou récursif si les choix de production et de consommation sont effectues l'un aprés l'autre, et indépendamment les uns des autres : le producteur maximise son profit (ou l'utilité espérée de son profit en situation d'incertitude). puis le consommateur maximise l'utilité tirée de sa consommation (ou l'utilité espérée). Le modele est non séparable lorsque labsence ou le mauvais fonctionnement de certains marchés (marché du travail. du capital, etc.) oblige à effectuer les choix de production et de consommation de façon simultanee. et en interaction l'un avec l'autre. Bien évidemment. la récursivité simplifie beacoup de calculs. Il est seulement dommage qu’elle ne corresponde pas a la réalité.

2. En effet. lorsque la production est vendue sur des marchés locaux, il est raisonnable de s'attendre a une forte corrélation négative entre l'offre et le prix : si l'offre est abondante, les prix baissent. Il n'en est pas de même lorsque le produit est vendu a létranger. dans des conditions telles que le prix mondial est insensible a une "bonne " ou " mauvaise " récolte dans tel ou tel pays particulier. En ce cas, pour le producteur d'un pays donné. il n'y a plus de relation entre sa récolte et le prix qu'il reçoit ; voir ci-aprés. SII. 2.

3. Lhypothese couramment admise est quen économie ouverte les prix et les quantités produites/ exportées ne sont pas corrélés, a moins que le pays producteur ne dispose d'un pouvoir de marché (situation monopolistique) tandis que én économie fermée. les prix et les quantités sont négativement corrélés. 
n'engendre pas une perte d'utilité. Cependant, Newbery et Stiglitz (1981) ont montré que même sous cette hypothèse de neutralité des producteurs face au risque, l'instabilité du revenu engendre une baisse de la production moyenne lorsque les prix et les quantités produites sont négativement corrélés. Ce cas de figure correspond à des marchés étroits, limités géographiquement (marchés locaux ou nationaux pour des produits vivriers) ou au cas de produits exportés lorsque le pays exportateur est en position dominante sur le marché mondial (Brésil pour le café, et peut-être la Côte-d'Ivoire pour le cacao). On peut encore l'appliquer au cas de l'ensemble des produits faisant l'objet d'échange international si l'on raisonne au niveau de l'offre et de la demande agrégées comme le font Newbery et Stiglitz.

Pour ces produits on peut alors montrer que l'instabilité réduit réellement l'espérance de recette'. Il en résulte une courbe d'offre apparente située au-dessus de la courbe de coût marginal. La différence entre le coût marginal et le prix moyen constitue une prime de risque pour le producteur (ou une marge markup) dont la valeur dépend des paramètres des fonctions d'offre et de demande. Elle représente en moyenne un profit pour le producteur, c'est pourquoi on dit que le risque " avantage " le producteur, de même que dans le cas du monopole, la différence entre la recette marginale et la recette moyenne représente un profit pour le monopoleur (voir fig. 1 en annexe).

En revanche, si l'on fait l'hypothèse que les prix et les quantités produites ne sont pas corrélés, cas de figure qui correspond à la majorité des produits d'exportation des pays africains pour lesquels ils sont preneurs de prix sur le marché mondial, l'espérance de profit est la même en situation risquée ou non risquée pour un producteur neutre par rapport au risque, de sorte que l'instabilité des prix et de la production ne modifie pas l'offre moyenne.

\section{Hypothèse d'aversion au risque des producteurs}

Il est en vérité peu probable que les producteurs soient neutres visà-vis du risque. Lorsque les producteurs ont de l'aversion pour le risque, celui-ci engendre un coût pour le producteur égal à la perte d'utilité lié à un revenu instable. Dans ce cas, le prix équivalent certain (prix certain qui engendre la même réponse de l'offre qu'en situation d'instabilité) dépend de la forme de la fonction d'utilité du producteur, de son degré d'aversion pour le risque, de l'élasticité de la demande, et

1. Le producteur neutre à l’égard du risque maximise $E(I I)-E(p q-w x)-(\hat{p} \bar{q}-w x)$. Pour une fonction de demande linéaire et un risque doffre multiplicatif. le prix équivalent certain (j) est égal à $\bar{p}\left(1 \ldots \xi_{i}^{2}\right)$. ¿ est l'élasticité de la demande évalué au prix moyen, et o l'écart type de l'aléa. 
de la corrélation entre les prix et les quantités'. En l'absence de corrélation entre prix et quantité (cas de la "petite nation ", ou du producteur petit par rapport au marché) le prix équivalent certain est toujours plus faible que le prix moyen, ce qui implique une production plus faible, et un prix moyen plus élevé, toutes choses égales par ailleurs, que dans le cas "certain » (voir fig. 1 en annexe).

S'il existe une corrélation entre prix et quantité (cas du producteur important de la "grande" nation, mais aussi cas du ménage qui consomme sa propre production, et pour qui la nourriture a d'autant plus de valeur qu'elle est rare), Newbery et Stiglitz (1981) ont montré qu'un accroissement de l'instabilité des prix entraîne une augmentation de la production lorsque les producteurs ont une forte aversion pour le risque, et une diminution de la production pour une aversion au risque modérée. En d'autres termes, les agents qui ont une forte aversion au risque, notamment les plus pauvres, augmentent leur effort et donc leur production pour éviter les risques extrêmes (famines...), tandis que les individus dont l'aversion est plus faible réduisent leur effort de production, car pour eux l'agriculture est une activité risquée qui n'est pas attractive.

\section{Risque et innovation technologique}

Par ailleurs, l'aversion pour le risque est un frein à l'adoption de nouvelles techniques de production qui, à long terme, exerce un effet négatif sur la croissance de l'offre agricole. L'innovation permet une augmentation du revenu moyen mais renforce généralement la variabilité du revenu. L'utilité attendue de l'utilisation de nouvelles techniques est alors plus faible pour les producteurs qui ont de l'aversion au risque. Il en résulte un taux d'adoption plus faible pour les producteurs ayant de l'aversion au risque et notamment pour les pauvres. De

1. Le producteur qui a de l'aversion au risque maximise $E[U(I I)]=E[U(p q \quad w x)]$ pour une fonction d'utilité séparable. E representant l'opérateur "espérance mathématique". et Lj une fonction dutilité " concave " (c"est-à-dire croissante, mais à " rendements decroissants"). Dans le calcul de lespérance de $\mathrm{L}$, les fortes valcurs du revenu dans les circonstances favorables "comptent moins" que les faibles valeurs dans les circonstances defavorables. De ce fait, le prix equivalent certain est inférieur a la moyenne du prix. Par exemple. Newbery et Stiglitz montrent que, pour une fonction dutilité a coefficient daaversion relative pour le risque constant, du type : $U$ $R$ son coefficient d aversion relative pour le risque, lorsque les prix et la production sont liés par une clasticité $\varepsilon$ du prix par rapport à l'offre. et que le risque d'offre est multiplicatif, avec un aléa lognormal (cela fait beaucoup dhypotheses?). le prix equivalent certain est donné par:

$$
\begin{aligned}
& \tilde{p}=\bar{p} \exp \left[-\left\{z+\frac{1}{2} \mathrm{R}(\varepsilon-1, z\} ;,\right]\right. \\
& \text { Cette expression est inferieure à pour des valeurs "normales" de }=\text { et de } \mathrm{R} .
\end{aligned}
$$


plus, lorsque l'innovation implique une dépense monétaire importante (par exemple l'achat d'engrais), les petits exploitants se heurtent à une contrainte de crédit généralement plus sévère que pour les grands exploitants, ce qui limite leur accès aux nouvelles techniques à degré d'aversion au risque équivalent ${ }^{1}$.

Diverses études de cas tendent à confirmer l'effet désincitatif du risque de prix sur l'offre de produits agricoles. Ainsi Berhman (1968), Guillaumont et Bonjean (1991) et Araujo (1995) ont fait apparaître, à travers des analyses économétriques de la réponse de l'offre de différentes cultures dans des pays d'Asie, d'Afrique et pour le Brésil, une élasticité négative de l'offre par rapport à une variable de risque de prix. Des analyses transversales sur les pays en développement ${ }^{2}$ ont également montré les effets désincitatifs de l'instabilité des prix sur l'offre agricole.

b) La réponse des ménages face au risque

Les marchés des produits vivriers dans les PVD sont généralement étroits et isolés, de sorte que les ménages paysans font face à des prix alimentaires instables et étroitement corrélés avec leur production. Dans ce cas, l'autoconsommation d'une partie ou de la totalité de la récolte par le ménage constitue une forme d'assurance contre le risque de prix : une chute des prix défavorable au producteur sera favorable au consommateur et inversement en cas de hausse des prix. Il en résulte que pour une même aversion au risque, le ménage paysan qui consomme sa propre production produit davantage qu'un producteur qui vend toute sa récolte, même si sa production reste toutefois réduite par rapport à une situation non risquée? . Ce comportement peut expliquer un paradoxe parfois rencontré dans les études empiriques : alors que les agents présentent de l'aversion au risque, la production proprement dite semble peu sensible au risque.

Toujours dans le cas où les prix à la consommation sont corrélés avec la production, la stratégie d'auto-assurance des ménages conduit ceux d'entre eux dont la part des produits vivriers dans le revenu est importante à produire davantage de vivriers que les autres, cela au détriment des productions de rente ${ }^{4}$.

Par ailleurs, Combes (1993) et Morduch (1992) ont montré, dans le cadre de modèles de consommation multipériodiques avec

1. Binswanger et Sillers, 1983. Ces auteurs montrent que des termes demprunt identiques condujsent à un risque plus élevé pour les petits exploitants.

2. Boussard et Gérard, 1994; Guillaumont et Combes, 1994 : Guillaumont et GuillaumontJeanneney, 1994.

3. Sadoulet et de Janvry, 1995

4. Fafchamps, 1992. 
revenu risqué, que les choix des ménages en matière d'épargne agissent sur le temps de travail alloué à l'activité agricole et sur les choix des spéculations.

\section{Conséquences du risque sur les ressources naturelles}

Les alternatives de "boom" et de dépression sont particulièrement nuisibles aux ressources naturelles qui sont alternativement surexploitées, puis laissées à l'abandon. Des exemples de tels phénomènes se rencontrent chez Ruf (1996), à propos des crises du cacao. Une période de "boom » conduit à exploiter un nouveau front pionnier, en détruisant la forêt vierge et en profitant des conditions naturelles idéales laissées par cette opération. L'exploitation, au début, est extensive, mais s'intensifie rapidement, en réponse aux incitations du marché qui n'a pas encore pris conscience de l'augmentation potentielle de l'offre. Au bout de quelques années, l'augmentation de l'offre fait baisser les prix, en même temps que les problèmes sanitaires liés à l'intensification apparaissent dans les plantations. Celles-ci sont bientôt ruinées, et cessent leur offre. Le prix alors remonte. Mais il est alors peu avantageux de reprendre la culture sur les anciennes plantations, encombrées de parasites. Il est bien plus avantageux de reprendre un nouveau front pionnier, et le cycle recommence. À chaque fois, des milliers d'hectares de forêt sont détruits de façon irréversible.

On peut probablement se passer de forêt vierge. Pourtant, de la même manière, les cycles du coton conduisent pendant un temps à étendre la culture et à l'intensifier dans des zones "limites ", actuellement par exemple au Tchad ou en Centre-Afrique. Puis la baisse des prix oblige les producteurs à réduire leurs apports de fertilisants. C'est là un comportement complètement rationnel, en application du principe de l'égalisation du coût marginal et du prix. La perte de production au début est faible, parce que le stock de matière organique du sol "fait tampon». Au bout de quelques années, cependant, ce stock est détruit, et sa disparition entraîne celle, irréversible, de toute la structure du sol, devenu impropre à la culture.

On a ici affaire à un défaut particulier dans le fonctionnement du marché, lié à sa "myopie ". On a dit que l'instauration de formes de propriété privée du sol seraient de nature à remédier à ce dysfonctionnement, parce que les producteurs alors raisonneraient à plus long terme, et intégreraient le maintien de la fertilité des sols dans leurs coûts. Ce serait peut-être vrai avec des taux d'intérêts moins élevés que ceux que nous connaissons aujourd'hui, et dans un contexte moins 
incertain. Dans la situation présente, avec des taux qui, pour les paysans, sont de l'ordre de 100 à $200 \%$, la valeur actuelle du patrimoine devient négligeable à un horizon de planification de deux ou trois ans, ce qui ferait perdre toute valeur incitative à la conservation du patrimoine naturel.

\section{LES CAUSES DE L'INSTABILITÉ}

L'instabilité des recettes de l'agriculteur résulte de celle des prix et des quantités produites, deux sources d'instabilité qui peuvent se renforcer ou se compenser. Un des apports de la théorie économique moderne dans l'analyse des sources d'instabilité en agriculture est de considérer la possibilité d'une instabilité endogène des revenus agricoles provenant des interactions dynamiques entre les prix et les quantités.

\section{L'instabilité exogène de l'offre}

L'instabilité engendrée par des perturbations exogènes aléatoires a longtemps été considérée comme la principale source de risque dans l'agriculture; elle provient essentiellement des perturbations climatiques, des maladies des plantes et des animaux, et d'autres calamités naturelles. L'agriculture dans les PVD est particulièrement exposée à ces risques pour de multiples raisons : faible développement des systèmes de production hors sol, impossibilité d'avoir recours à l'irrigation, sous-utilisation des produits phytosanitaires, etc. De telles perturbations s'apprécient différemment à différentes échelles.

Il est généralement admis que l'instabilité de l'offre est plus grande dans un marché étroit ou de faible dimension géographique'. Lorsque le rayon d'action du marché augmente, si les aléas sont distribués uniformément et indépendamment les uns des autres sur la surface géographique, alors la loi des grands nombres assure que la variabilité relative de l'offre devrait décroître. Mais il faut pour cela que la corrélation entre les aléas en deux points différents soit nulle ou négligeable, ce qui est sans doute vrai pour des points éloignés, et manifestement faux pour des points situés à quelques kilomètres l'un de l'autre. Cela pose le problème de la «distance de corrélation » de 
ces aléas, la distance au-delà de laquelle la corrélation devient négligeable. Des études agro-météorologiques' ${ }^{1}$ semblent montrer qu'elle est de l'ordre de $100 \mathrm{~km}$. Il reste à déterminer dans quelle mesure des aléas survenant sur la surface correspondante sont en mesure de perturber les marchés et, surtout, quelle sorte de marché : on peut en effet s'attendre à observer dans ce cas des fluctuations des marchés de sous-préfectures, mais sans doute pas des marchés nationaux ou internationaux.

\section{Les variations des prix}

Dans un cadre statique, le lien entre l'instabilité des prix et celle de l'offre dépend du caractère échangeable ou non échangeable du produit et donc du degré d'intégration du marché national au marché mondial.

En économie fermée, les variations des prix des productions agricoles et des quantités correspondantes sont corrélées négativement. De plus, les fluctuations de l'offre engendrent des fluctuations de prix qui tendent à être plus que proportionnelles aux variations de quantités en raison de la rigidité de la demande (loi de $\mathrm{King})^{2}$. Il en résulte que les revenus des producteurs sont plus grands quand l'offre est faible. Par ailleurs, les fluctuations de revenu sont en général moins grandes, en valeurs absolues, que les variations de prix.

En économie ouverte, au contraire, les prix sont une donnée exogène pour les petits pays importateurs ou exportateurs. L'instabilité des prix est alors "importée " et dépend des conditions d'offre et de demande sur les marchés mondiaux, du cours des monnaies, etc. L'instabilité des prix et des quantités ont alors peu de chances de se compenser mais tendent au contraire à se renforcer ${ }^{3}$.

Ici encore se pose la question de savoir si un élargissement du marché est de nature à le stabiliser. Si les «chocs» sur les prix sont tous d'origine aléatoire, commandés par des aléas uniformément répartis sur la surface du globe, et indépendamment les uns des autres, alors, comme pour les aléas physiques, un élargissement du marché est de

1. Martineu et Tissot. 1993 ; Chtara, 1996 : Nubukpo. 1994.

2. Soit a lélasticité-prix de la demande. $\mathrm{Si}$ = est inférieur à 1 en valeur absolue. une variation de quantité de $1 \%$ produira une variation de prix de plus de $1 \%$. On en déduit que l'instabilité des prix est plus que proportionnelle à celle de la production.

3. En effet. si lon mesure l'instabilité du revenu par la variance de ce revenu exprimé en logarithmes, on a : var $(\log Y) \cdots \operatorname{var}(\log P)+\operatorname{var}(\log Q)+2 \operatorname{cov}(\log P, \log Q)$. Pour un petit pays preneur de prix. cov (P. Q) est nulle et la variance du revenu est égale a la somme de la variance des prix et des quantités. 
nature à le stabiliser. Cet argument a été employé pour culpabiliser l'UE et les États-Unis, et les accuser de déstabiliser les marchés mondiaux, en y déversant leur instabilitél.

\section{Les interactions dynamiques entre prix et offre}

En raison des délais entre les plantations et la récolte, les producteurs se trouvent confrontés à une autre source d'instabilité liée aux erreurs d'anticipation des prix futurs. Le schéma le plus classique dans lequel les erreurs d'anticipation conduisent à une instabilité endogène des prix et de l'offre est celui du Cobweb. La référence de base en la matière est Ezekiel (1938), suivie de plusieurs études postérieures sur "qui bénéficie " et "qui perd" au jeu de ces fluctuations?.

Pour cette école de pensée, l'instabilité provient des erreurs d'anticipation des agents (producteurs, ou spéculateurs). Pour certaines valeurs des paramètres des fonctions d'offre et de demande, ces erreurs d'anticipation donnent lieu à des phénomènes chaotiques, c'est-à-dire des fluctuations qui ne sont jamais périodiques (même s'il est toujours possible de croire qu'il existe une période, un "cycle " pendant souvent assez longtemps) et de ce fait imprévisibles". Le fait que la demande soit rigide rend les phénomènes de cette sorte particulièrement fréquents en agriculture.

L'instabilité des prix agricoles peut donc être de nature exogène, liée à des perturbations aléatoires de l'offre ou de la demande, ou de nature endogène, née du jeu des marchés et notamment des mécanismes d'anticipation des prix. Il est essentiel de bien distinguer ces deux types d'instabilités, dont les conséquences sont tout à fait différentes. Face à une instabilité d'origine exogène, l'élargissement des marchés agricoles devrait permettre de stabiliser les prix. De plus, dans un tel contexte, la spéculation est bénéfique : les spéculateurs achètent quand le prix est bas, ce qui rééquilibre le marché, pour revendre quand le prix est haut, ce qui l'empêche de monter encore plus. Mais dans un contexte d'instabilité endogène, ces phénomènes ne jouent plus : les marchés se synchronisent. Les spéculateurs, souvent, se trompent et, faute d'information pertinente, ils opèrent à contretemps. Dans ce cas, non seulement ils se ruinent personnellement, mais encore, ils aggravent la situation de tout le monde. Ainsi, Ravallion (1987), Azam et Bonjean (1995), Deaton et Laroque (1992) ont montré qu'en économie

1. Bale et Lutz. 1979.

2. Waught. 1944; Massel. 1969, Oi, 1961

3. Cf. Boussard, 1996. 1994. 1993: Chavas et Holt. 1993 : Araujo Bonjean. 1997. 1998. 
fermée les interventions des opérateurs stockeurs en information imparfaite peuvent conduire à une instabilité des prix plus grande que dans un équilibre d'anticipations "rationnelles", c'est-à-dire sans erreur.

D'une façon générale, dans le cas d'une instabilité endogène, le marché ne peut pas lui-même conduire à une réduction de l'instabilité, à moins d'en corriger les dysfonctionnements. Cette remarque est à la base de la justification de l'intervention de l'État sur les marchés agricoles. Une telle intervention, dans ce contexte, doit avoir pour but de rétablir artificiellement sur les marchés réels l'équilibre qui aurait dû s'établir si ceux-ci avaient fonctionné comme le veut la théorie idéale des marchés parfaits.

III. LA GESTION DE L'INSTABILITÉ

Nous avons vu plus haut (II.2) que le marché n'assure généralement pas une stabilisation "spontanée " des revenus agricoles. Même lorsque les producteurs vendent uniquement sur le marché intérieur où joue "la loi de l'offre et de la demande ", les variations de prix ne compensent généralement pas les fluctuations de l'offre, bien que ce mécanisme puisse aboutir à réduire les fluctuations de revenu par rapport aux fluctuations de quantité. Les fluctuations des revenus agricoles sont donc susceptibles d'engendrer un risque de consommation pour les ménages paysans. Or pour ces ménages, le coût du risque lié à l'instabilité des revenus agricoles dépend in fine de ses conséquences sur la variabilité de leur consommation'.

Pour les producteurs qui peuvent épargner les bonnes années et emprunter les mauvaises années, le coût du risque est réduit. Cependant, les paysans des pays en développement ont généralement peu de moyens pour emprunter, et donc peu de moyens de se protéger contre l'instabilité de leur revenu. En effet, en raison des dysfonctionnements du marché du crédit, des taux d'intérêt élevés, de la quasi-absence de marché de l'assurance et des difficultés de stockage, les ménages agricoles, et notamment les plus pauvres, ne sont généralement pas en mesure d'opérer un lissage de leur consommation dans le temps. Dans ces conditions, l'instabilité du revenu engendre un risque de consommation. Ainsi, du fait d'un accès limité au marché des capitaux, les 
paysans sont incités à réduire l'instabilité de leur revenu en diversifiant leurs activités ou en partageant le risque avec d'autres agents. L'État peut aussi prendre en charge l'instabilité des revenus agricoles à travers la création de systèmes d'assurance récolte ou de mécanismes de stabilisation des prix.

\section{Gestion du risque au niveau du ménage}

Selon la théorie du revenu permanent, en l'absence de marché de l'assurance, le producteur dont les revenus sont instables épargne les recettes exceptionnelles dégagées les bonnes années de façon à compenser les chutes futures de revenu. De plus, l'incertitude attachée aux revenus futurs conduit le producteur, s'il est prudent au sens de Kimball (1990)', à développer une épargne de précaution qui augmente avec l'instabilité de son revenu². Ainsi, les ménages peuvent en principe se protéger grâce à leur épargne contre les chutes aléatoires de leur revenu. Cependant, si l'accès au crédit est restreint, les ménages les plus pauvres qui ont épuisé leurs actifs peuvent être contraints à réduire leur consommation ${ }^{3}$. De plus, les ménages, et notamment les plus pauvres, restent vulnérables aux chocs de revenu lorsque ceux-ci ont un caractère permanent (destruction des plantations par le feu...) ou lorsqu'il existe une autocorrélation intertemporelle des revenus (par exemple, quand une mauvaise année en entraîne une autre, en empêchant la mise en ouvre des techniques nécessaires) ${ }^{4}$. Enfin, le risque lié au taux de rendement des capitaux investis, certainement important en milieu rural, décourage l'épargne de précaution ${ }^{5}$. Les stratégies individuelles d'épargne ne sont donc que des substituts très imparfaits à l'assurance ; néanmoins, les études empiriques tendent à montrer que les ménages ruraux épargnent une fraction importante de leur revenus transitoires ce qui leur permet de réduire significativement l'instabilité de leur consommation par rapport à celle de leur revenu'.

Les producteurs qui, en raison des défaillances de marchés, ne peuvent pas opérer un lissage parfait de leur consommation peuvent aussi réduire l'instabilité de leur revenu en diversifiant leurs productions en

1. Un individu est prudent si l'utilité marginale de son revenu est convexe (voir ci-après).

2. Guillaumont et al. 1998.

3. Deaton, 1990. Ces marchés sont généralement peu développés en milieu rural et les taux d'intérêt élevés en raison de l'importance des coûts de fonctionnement. de sorte que le recours au crédit ne peut ètre considéré à l'heure actuelle comme un moyen efficace de lissage de la consommation.

4. Deaton, 1990. 1991 ; Besley, 1995.

5. Combes, 1993.

6. Wolpin, 1982 ; Bevan, Collier et Gunning, 1989 ; Paxson, 1992 ; Combes, 1993. 
fonction des risques associés à chaque spéculation. D'une façon générale, la prise en compte de l'interdépendance des choix de production et de consommation, qui découle notamment de la présence d'une contrainte de liquidité, conduit à des plans de production dans lesquels la part des productions risquées est moins importante et le nombre des productions plus grand, et à une diversification spatiale des activités'.

Par ailleurs, nous avons vu plus haut que le développement des productions autoconsommées constitue dans certains cas une forme d'auto-assurance des ménages contre le risque de prix. Ces deux stratégies de réduction du risque, diversification des productions et augmentation des cultures vivrières, sont généralement coûteuses dans la mesure où la diversification prive les producteurs des gains de la spécialisation, et où les productions vivrières sont généralement des activités à plus faible rendement ${ }^{2}$.

D'autres réponses possibles face au risque de revenu consistent à partager le risque avec d'autres agents soit en recourant au crédit informel ${ }^{3}$, à la solidarité familiale ou villageoise ${ }^{4}$, soit en recherchant des revenus non agricoles, éventuellement à travers la migration... Les producteurs peuvent également, à travers le choix d'un contrat de travail, réduire l'instabilité de leur revenu. Ainsi, le travail salarié et le métayage sont deux formes de contrat permettant de transférer une partie ou la totalité du risque sur un agent, le propriétaire, dont l'aversion au risque est plus faible. La contrepartie de cette réduction du risque est alors une productivité inférieure en raison des effets désincitatifs de ces contrats 5 .

Ainsi, en présence de marchés incomplets, comme c'est le cas dans les pays d'Afrique subsaharienne, les stratégies d'épargne, de diversification des activités et de diffusion des risques permettent aux ménages agricoles de s'assurer partiellement contre le risque lié à l'instabilité de leur revenu. Cependant, les stratégies d'auto-assurance des ménages sont à la fois partielles et coûteuses : elles conduisent à une diminution et à un moindre lissage intertemporel de leur consommation, voire à une diminution de leur revenu moyen ${ }^{6}$. Ainsi, une prise en charge par l'État ou par des organismes intermédiaires de l'instabilité des revenus agricoles, à travers la création de systèmes

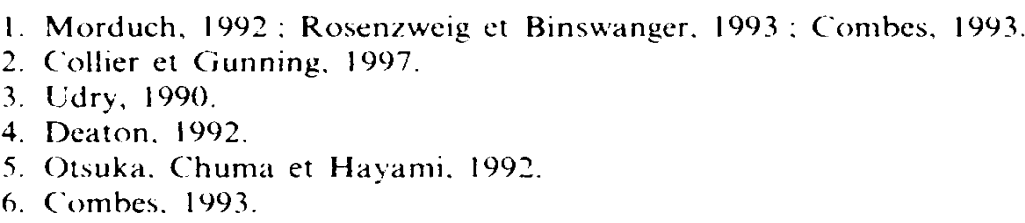


d'assurance récolte ou de mécanismes de stabilisation des prix, peut être justifiée, notamment du point de vue des plus pauvres qui n'ont ni la possibilité d'épargner ni celle de diversifier leurs activités sans mettre en danger leur subsistance.

\section{L'assurance récolte et l'assurance revenu}

Présentée comme une panacée dans certains milieux américains ou canadiens, l'assurance récolte est vivement critiquée par les économistes professionnels'.

Une des difficultés auxquelles se trouve confronté un système d'assurance récolte tient à la corrélation des risques agricoles au niveau d'une région ou d'un pays: les catastrophes météorologiques de grande ampleur telles que les sécheresses frappent parfois de vastes zones géographiques, ce qui accroît considérablement le risque de faillite de l'organisme d'assurance. Mais surtout, un système d'assurance récolte, comme tout autre type d'assurance mais sans doute bien plus dans l'agriculture qu'ailleurs, se heurte au problème de la "sélection contraire " et de l' "aléa moral " liés aux asymétries informationnelles.

Le phénomène de sélection contraire consiste en ce que, du fait que la prime d'assurance est nécessairement calculée sur la base d'une moyenne, les individus ayant des risques faibles mais non nuls trouvent la prime trop élevée ${ }^{2}$, et sont éjectés du système. Ce phénomène renforce l'exclusion des individus les plus pauvres qui n'ont pas la capacité de s'assurer, même au taux moyen.

L' "aléa moral » résulte du fait que l'existence même du contrat d'assurance conduit le titulaire à prendre moins de précautions pour éviter les sinistres. Habituellement, les compagnies résolvent ce problème en imposant à leurs clients des dispositifs particuliers, par exemple des serrures perfectionnées pour les assurances contre le vol. Mais cela suppose que l'on soit capable de vérifier l'exécution de la clause en question d'une façon simple et peu coûteuse. C'est le cas pour les serrures d'un certain modèle dans les appartements parisiens, mais ce n'est pas du tout aussi facile avec une multitude de petits exploitants dispersés géographiquement ${ }^{3}$. Il est toutefois possible de réduire les coûts de gestion et l'incidence de l'aléa moral en calculant les indemnités sur la base de la chute de la production d'un ensemble homogène

1. Hueth el al. 1994.

2. Pour une prime dassurance calculée sur la base du taux moyen doccurrence du préjudice concerné. seuls sont incités à s'assurer les individus les plus exposés au risque. d'où une sélection "contraire " des individus désirant s"assurer.

3. Binswanger et Rosenzweig, 1986. 
d'exploitations d'une même région par rapport à une année moyenne'. Toutefois, ce système pénalise les agriculteurs les plus performants.

$\mathrm{Au}$ total, un mécanisme d'assurance récolte ne peut offrir qu'une garantie limitée pour les producteurs, notamment pour les plus pauvres qui risquent d'en être exclus. Ce mécanisme ne permet pas non plus de protéger les exploitants contre le risque de prix qui peut être au moins aussi important que le risque d'offre.

Face à cette limite de l'assurance récolte et à la suite de l'échec des politiques de garantie de prix dans les pays développés, on a envisagé la mise en place d'un mécanisme d'assurance " revenu " sous forme de «marché d'options» qui permettrait aux petits exploitants de se couvrir contre le risque de prix ou de récolte de la même manière que les gros opérateurs publics ou privés le font en intervenant sur les marchés à terme. Dans ce système, les producteurs peuvent acheter des options et recevoir une prime lorsque le prix ou le rendement sont inférieurs au prix et au rendement d'exercice. Toutefois, un tel système ne peut fonctionner sans la participation de l'État qui devrait notamment apporter une subvention de façon à ce que ce mécanisme soit accessible aux plus pauvres ${ }^{2}$. On retombe alors dans la logique des subventions et des interventions directes.

\section{La stabilisation des prix}

La stabilisation des prix par l'État peut être un objectif souhaitable de politique économique lorsque les producteurs n'ont pas les moyens de se protéger contre l'instabilité de leur revenu, et lorsque la stabilisation des prix est de nature à stabiliser leur revenu. La stabilisation exercée à travers la constitution d'une épargne publique est alors un substitut à l'épargne privée particulièrement désirable pour les petits producteurs proches du seuil de subsistance ${ }^{3}$. Il reste à définir les fonctions de l'organisme régulateur, le niveau autour duquel le prix doit être stabilisé et d'éventuels garde-fous permettant d'assurer la pérennité du système.

a) Les fonctions de l'organisme stabilisateur

Selon la nature du produit, produit vivrier destiné au marché national ou produit d'exportation, l'organisme stabilisateur peut être amené ou non à gérer physiquement le produit à travers la constitution de stocks régulateurs.

1. Newbery. $1989 \mathrm{~h}$.

2. Guillaumont et al. 1998

3. Combes. 1993. 
Les caisses de stabilisation pour les cultures d'exportation

Conçues pour gérer l'instabilité des prix des produits agricoles d'exportation, les caisses de stabilisation ou marketing board sont souvent devenues des organismes parafiscaux, et les fonds collectés ont davantage servi à financer les dépenses de l'État qu'à assurer le soutien des prix intérieurs en période de chute. De plus ces organismes, auxquels on a reproché des effectifs pléthoriques, ont eu parfois à gérer physiquement les produits et ont obtenu un monopole sur la commercialisation extérieure dont il est résulté une grande inefficacité. Il n'en demeure pas moins qu'à condition de donner à ces organismes suffisamment d'indépendance vis-à-vis de l'État et d'en limiter les compétences à la seule stabilisation des prix, ils pourraient assurer une stabilisation efficace des prix domestiques des produits d'exportation pour un coût d'opération relativement réduit'. Leur mission devrait se limiter à stabiliser les prix à la production par un mécanisme de taxation et de subvention sans que les caisses soient impliquées dans la commercialisation des produits. Les fonds de stabilisation devraient être placés en dehors des circuits des Trésors publics et leur utilisation réservée aux fins de stabilisation des prix agricoles.

\section{Les stocks régulateurs}

Dans le passé, de nombreux pays ont mis en place des offices publics chargés de contrôler et de réguler le commerce et les prix des produits vivriers en achetant et en vendant lorsque le prix devient inférieur à un prix plancher ou supérieur à un prix plafond. Ces organismes étaient notamment chargés de lutter contre les amples fluctuations saisonnières des prix des produits vivrières mais aussi parfois de gérer un stock national de sécurité, les importations et les subventions à la consommation.

La principale difficulté à laquelle se heurte un tel mécanisme est la détermination du niveau optimal de stockage. En réalité, le volume des stocks est régi par une loi de probabilité de "marche au hasard " qui implique que, au bout d'un certain temps, le stock est nul ou la capacité de stockage saturée avec une probabilité voisine de $1^{2}$. Pour cette raison, les opérations de stockage/déstockage aboutissent à des crises plus espacées mais plus profondes que celles qui résultent du libre jeu des marchés.

1. Guillaumont et Guillaumont, 1990.

2. Newbery et Stiglitz, 1981 ; Deaton et Laroque, 1992. 
Ces offices ont, avec les programmes d'ajustement et l'ouverture des économies, vu leurs missions réduites pour généralement se limiter à la gestion d'un stock de sécurité dont le rôle n'est plus d'assurer la stabilisation des prix mais d'éviter les situations extrêmes de pénuries. Il semble peu opportun aujourd'hui de réintroduire de tels mécanismes de stabilisation en raison principalement de leurs coûts de fonctionnement, de leur inefficacité à contrôler une part importante du marché et des multiples fraudes qu'ils occasionnent.

b) A quel niveau faut-il stabiliser les prix des cultures d'exportation?

Une des raisons de l'échec des mécanismes de stabilisation, au niveau national ou international, est la confusion qui a parfois été faite entre les objectifs de stabilisation et de soutien des prix ; lorsque ces mécanismes ont été utilisés pour lutter contre la tendance à la baisse des prix, ils se sont rapidement épuisés.

Un mécanisme de stabilisation viable ne peut prendre en charge que les fluctuations transitoires des prix autour de leur valeur moyenne ou tendancielle, et ne devrait pas servir à retarder un ajustement face à une modification structurelle du marché sous peine d'engendrer une allocation inefficiente des ressources et des coûts de gestion rédhibitoires. Autrement dit, les chocs temporaires de prix peuvent faire l'objet d'une gestion contracyclique, tandis que les chocs qui ont un caractère permanent nécessitent un ajustement. Le problème est alors de déterminer le caractère permanent ou temporaire des chocs qui affectent les prix agricoles, autrement dit la nature déterministe ou stochastique de la tendance des prix'.

Dans le cas où les prix fluctuent de façon aléatoire autour d'une tendance déterministe, une stabilisation totale ou partielle des prix (à l'intérieur d'une fourchette de prix) est d'autant plus souhaitable que les coûts d'ajustement dans le secteur agricole sont élevés et les délais de réponse de l'offre sont longs ${ }^{2}$. Compte tenu de la réponse de l'offre à l'instabilité des prix, ceux-ci doivent être stabilisés à un niveau inférieur à leur moyenne, correspondant à l'offre moyenne (cf. ci-dessus, $\S \mathrm{I} .2 .1)$.

Cependant, si la principale source de risque est l'instabilité endogène de l'offre née des erreurs d'anticipation des producteurs, une politique de stabilisation des prix, en permettant d'atteindre un équilibre d'anticipations rationnelles, permettrait, en théorie, d'accroître

1. Newbery et Stiglitz, 1981 ; Deaton et Laroque, 1992.

1. Le soutien des prix en période de baisse transitoire des cours doit permettre de maintenir des plantations qui, si elles étaient abandonnées, ne pourraient être remise en culture rapidement au moment du retournement des cours. 
l'efficience et de réduire l'instabilité des revenus; ce n'est cependant pas le seul moyen ni le plus efficace de corriger les erreurs d'anticipation. Des actions en matière de collecte et de diffusion de l'information sur les marchés par les pouvoirs publics pourraient être aussi efficaces et moins coûteuses.

c) Une garantie de prix limitée par des quotas

La stabilisation des prix au producteur risque de se heurter à un obstacle lié aux caractéristiques de la fonction de production en agriculture. Avec une fonction de production homogène et de degré 1 , en l'absence de facteur fixe limitant de façon ultime la croissance de l'offre (c'est le cas, souvent, en Afrique, où des terres vierges sont encore disponibles), l'achat par l'État, à prix fixe, de toute quantité offerte sur le marché conduit à une offre infinie', et donc à la faillite de la "caisse de stabilisation ». Dans ce cas, ou de façon plus générale pour limiter le risque de perte de l'organisme stabilisateur, il peut être opportun de limiter la garantie de prix à un quota de production déterminé à l'avance.

Avec ce système, chaque agriculteur dispose du " droit de produire " une certaine quantité à un prix garanti par l'État. S'il produit plus, suivant les cas, il peut écouler le surplus au prix du marché (cas du sucre dans la (EE), ou subir des pénalités allant jusqu'à la confiscation du produit (cas du lait dans la CEE). Le prix garanti est normalement assez attractif pour que toute les quantités correspondantes soient produites. On obtient ainsi un moyen efficace de garantir un certain niveau de sécurité alimentaire par la production intérieure. Par ailleurs, le producteur a toujours intérêt à diminuer ses coûts. Au Québec, les quotas laitiers ont permis de diviser les coûts de production par deux en quinze $a^{2} s^{2}$. Une simulation récente sur le marché du sucre $^{3}$ montre qu'un tel système est capable d'augmenter le bien-être global.

En revanche, le système présente certains inconvénients : il "gèle " les structures de production, et rend difficile les adaptations à de nouvelles technologies. C'est pourquoi (contrairement à ce qui se passe dans la CEE pour les quotas laitiers) il est souhaitable qu'il soit négociable $^{4}$, comme en témoigne l'expérience du Québec. Par ailleurs, sa mise en place est difficile, parce qu'il faut une administration efficace et peu corrompue. L'attribution des quantités initiales pose de sérieux

1. Boussard, 1993, 1994, 1995.

2. Gouin et Morrisset, 1992.

3. Boussard et Piketty, 2000.

4. Boussard, 1995. 
problèmes d'équité. Enfin, pour éviter les fraudes, il faut que le produit transite par un point de passage obligé au cours de son traitement. C'est sans doute le cas du cacao ou du coton, mais pas des cultures vivrières en Afrique.

\section{Les marchés à terme}

Les marchés à terme offrent deux avantages distincts aux producteurs. D'une part ils leur permettent d'éviter le risque de prix (fonction de transfert du risque). Ainsi, à court et moyen termes les marchés à terme garantissent un prix pour au moins une partie de la récolte. Mais le producteur peut aussi spéculer sur le prix futur plutôt que de se couvrir contre le risque. D'une façon générale, les marchés à terme apportent au producteur une meilleure assurance revenu que celle offerte par la stabilisation des prix, le producteur étant libre de choisir son niveau optimal de couverture alors que la stabilisation des prix impose une couverture totale de la production. D'autre part, si ces marchés sont efficients, ils permettent de lever les erreurs de prévision sur les prix futurs (rôle informationnel). En améliorant l'efficience informationnelle des marchés, ils permettent de se rapprocher d'un équilibre d'anticipations rationnelles et de limiter les fluctuations de prix dues à des phénomènes de type cobweb.

Cependant, le rôle des marchés à terme est nécessairement limité. Ils ne protègent que contre le risque de prix. Du fait du caractère aléatoire de la production, le producteur ne connaît pas par avance la quantité de produit qu'il doit assurer ; les marchés à terme ne peuvent donc pas constituer des substituts à une assurance parfaite. D'autre part, les marchés à terme ne suppriment pas le risque de prix ; les prix sur les marchés à terme sont aussi variables, sinon plus, que les prix spot. De plus, le terme des contrats est généralement court, inférieur ou égal au temps de gestation des cultures (la plupart des contrats sont de six mois ou moins). Dans le meilleur des cas ils ne permettent donc que d'éliminer le risque de prix intra-annuel, mais le risque de prix subsiste d'une année sur l'autre. Les marchés à terme ne permettent donc pas de stabiliser le revenu du producteur sur plusieurs années ni de le protéger contre le risque lié aux fluctuations de sa consommation'. Enfin, les coûts de transaction associés à l'utilisation des marchés à terme tendent à être élevés notamment en raison de

1. Toutefois, la couverture intra-annuelle apportèe par les marchés à terme peut être désirable pour le producteur qui finance ses intrants à credit. 
l'importance du volume minimum des transactions souvent supérieur à la quantité optimale pour le producteur.

Si les petits producteurs ne peuvent avoir un accès direct aux marchés à terme, ils peuvent en bénéficier indirectement à travers des contrats passés à prix fixe avec des sociétés de négoce ou un organisme de stabilisation. De telles structures, elles, pourront recourir aux marchés à terme pour se couvrir contre le risque lié aux contrats passés avec les petits producteurs. Il est à noter que les interventions massives des caisses de stabilisation sur des marchés étroits risquent d'être déstabilisantes.

\section{Les "contrats de culture" et l'intégration verticale}

Indépendamment des marchés à terme organisés dans les grandes places internationales, il faut noter que les «contrats de culture » et les mécanismes d" "intégration verticale " constituent des sortes de contrats à terme qui stabilisent les prix payés aux producteurs sur le court terme, tout en assurant une rémunération (en général plutôt faible) de leur travail. Il existe sur ce sujet une abondante littérature française du début des années soixante, malheureusement injustement oubliée à l'heure actuelle'. L'intégration verticale présente l'avantage supplémentaire de faire bénéficier le producteur d'un suivi technique permanent de la part de l'intégrateur. On obtient de la sorte en particulier une assurance de qualité et de conformité du produit qu'on a bien du mal à obtenir autrement. Le système du " coton CFDT » est une parfaite illustration des avantages de ce type d'organisation dans le contexte africain ${ }^{2}$

Bien entendu, comme les marchés à terme, les contrats d'intégration verticale, s'ils suppriment le risque du producteur au moins dans un cadre intra-annuel, tout en lui laissant la charge du coût correspondant sous forme de baisse du prix moyen qui lui est payé, n'agissent en principe pas sur l'instabilité des prix au consommateur et ses effets nuisibles. Toutefois, dans la mesure où les firmes intégratrices sont de grande dimension et disposent d'un certain pouvoir de monopole, il arrive qu'elles soient en position d'imposer au marché un certain niveau de stabilisation, à un prix élevé il est vrai. Dans les pays développés, on voit ainsi les grandes centrales de distribution s'efforcer de «lisser " les prix de détail, même pour des produits très "volatils » comme le café, afin d'éviter à leur clientèle des variations de prix qu'elle ne comprendrait pas, et qui risqueraient d'entraîner une désaffection vis-à-vis de

1. Cf., par ex. Lebosse et Ouisse, 1974, ou Le Bihan, 1960.

2. Lele, 1990. 
« la marque ». Il n'est pas sûr que le procédé, efficace pour des produits à faible coefficient budgétaire dans les pays riches, soit transposable aux denrées de base dans les pays en développement.

\section{CONCLUSION}

L'analyse théorique a montré que lorsque les marchés sont incomplets (absence de marchés à terme offrant des contrats de long terme, absence de marchés de l'assurance), l'équilibre de concurrence parfaite et d'anticipations rationnelles n'est généralement pas efficient. Bien qu'une intervention publique soit alors justifiée, il n'est pas clair qu'une politique de stabilisation des prix permette d'accroître l'efficience économique. Il peut être préférable de modifier l'environnement structurel des producteurs en améliorant le fonctionnement des marchés défaillants (marchés du crédit, de l'assurance...) plutôt que de stabiliser les prix dans un cadre de marchés incomplets. La justification théorique d'une politique de stabilisation des prix internationaux des produits agricoles demeure donc fragile.

Cependant, les pays d'Afrique au sud du Sahara présentent des caractéristiques qui peuvent justifier une prise en charge par l'État de l'instabilité des revenus tirés des exportations agricoles à travers la stabilisation des prix à la production. D'une part, les défaillances des marchés de l'assurance et du crédit sont patentes et les conditions de restauration ou d'implantation de ces marchés ne sont pas évidentes, en raison notamment du coût élevé de leur fonctionnement. Les producteurs n'ont donc généralement pas la possibilité de se protéger efficacement contre le risque lié à l'instabilité de leur revenu. Les stratégies d'épargne, de diversification des activités et de diffusion des risques n'apportent qu'une assurance partielle contre le risque et ont un coût social parfois élevé. D'autre part, ces pays sont en règle générale preneurs de prix sur les marchés mondiaux des produits agricoles. L'instabilité des prix mondiaux est donc pour eux une source additionnelle de risque qui s'ajoute au risque de production. Dans ce cas, la stabilisation des prix permettrait de stabiliser le revenu des producteurs et d'accroître l'offre des produits stabilisés. De plus la stabilisation des prix payés aux producteurs de cultures d'exportation n'implique pas la mise en place de stocks régulateurs ni la gestion physique des produits par les pouvoirs publics, ce qui permet d'alléger significativement le coût de la stabilisation. Il importe toutefois de ne pas sous-estimer les coûts de fonctionnement d'un organisme de stabilisation et les difficultés liées à la définition d'un prix de référence. L'expérience passée du 
fonctionnement des caisses de stabilisation a également montré les dangers d'une mauvaise gestion des fonds de stabilisation qui a pu conduire dans certains cas à une aggravation des déséquilibres macroéconomiques. Il conviendrait donc peut-être de privilégier d'autres mécanismes de réduction du risque en poussant plus loin l'analyse des systèmes d'assurance et d'intégration verticale.

ANNEXE

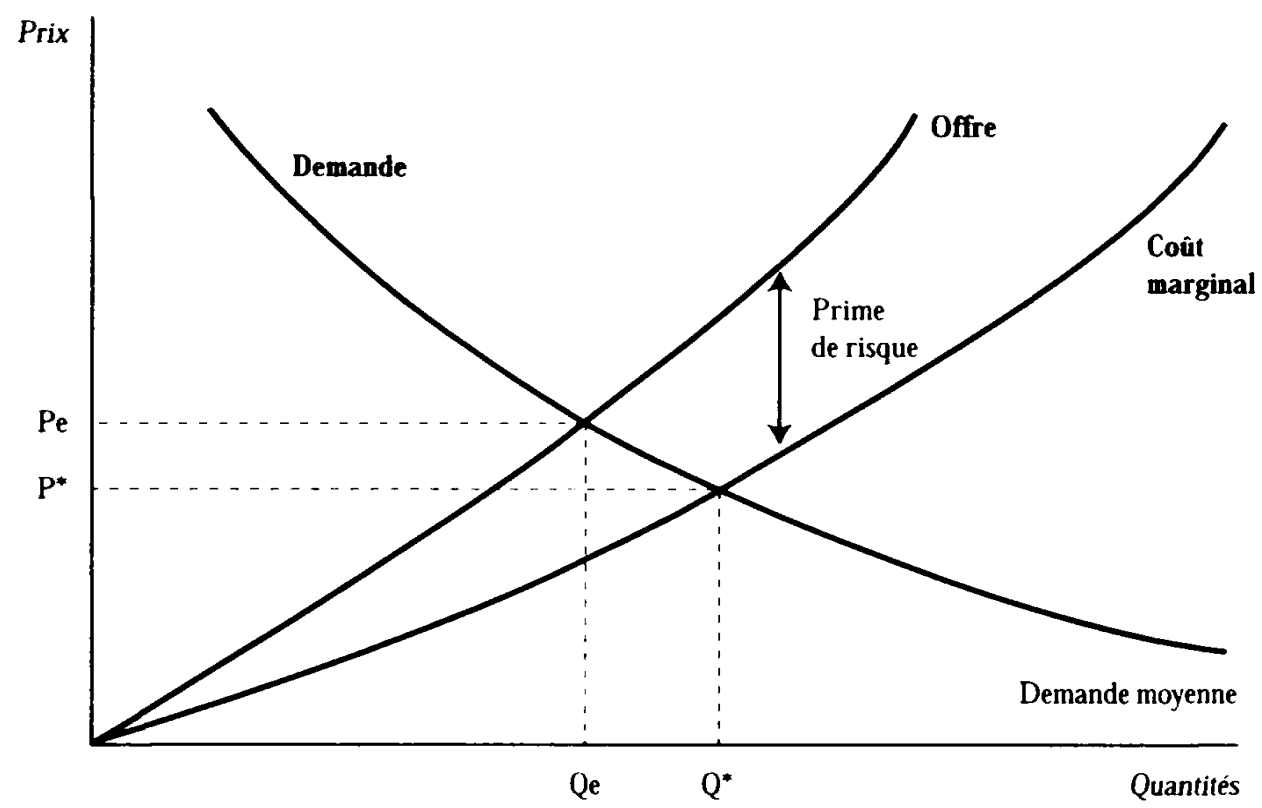

Fig. 1. - Offre en présence de risque

En présence de risque, le producteur choisit un niveau d'offre espéré Qe en égalisant son coût marginal avec l'équivalent certain du prix du prix moyen $(P)$. Comme cet équivalent certain est inférieur au prix moyen, la différence étant la "prime de risque", il faut ajouter cette prime au coût marginal pour obtenir la courbe d'offre. L'équilibre moyen au lieu d'être en $\left(\mathrm{Q}^{*}, \mathrm{P}^{*}\right)$ se situe maintenant en $(\mathrm{Q} e, \mathrm{P} e)$ avec une quantité offerte plus faible et un prix moyen plus élevé.

\section{RÉFÉRENCES}

Araujo, C. (1995), Les producteurs brésiliens et l'instabilité des prix : différences de comportement entre le Nord et le Sud, Canadian Journal of Agricultural Economics, 43 (1), 443-461.

Araujo Bonjean, C. (1997), Les marchés mondiaux des produits agricoles sont-ils efficients?, Économie rurale, $\mathrm{n}^{\circ} 243$, janvier-février. 
Araujo Bonjean, C. (1997), Termes de l'échange : de la baisse tendancielle au chaos, Revue d'économie du développement, 1, 105-128.

Azam, J.-P. et Bonjean, C. (1995), La formation du prix du riz: théorie et application au cas d'Antananarivo (Madagascar), Revue économique, 48 (4), juillet.

Badillo et Dalloz (1985), Marché, spéculation, stabilisation, Paris, Economica.

Bale, M. et Lutz, E. (1979), The Effects of Trade Intervention on International Price Stability. American Journal of Agricultural Economics, 61 (3), 512-516.

Behrman, J. R. (1968), Supply response in underdeveloped agriculture, Amsterdam, North-holland Publishing Compagny.

Besley, T. (1995), Savings, credit and insurance, in J. Behrman et T. N. Srinivasan (éd.), Handbook of Development Economics, vol. 3A, NorthHolland, Elsevier Science.

Bevan, D. L., Collier, P. et Gunning, J. W. (1987), Consequences of a commodity boom in a controlled economy : Accumulation and redistribution in Kenya 1975-1983, World Bank Economic Review, 1, 489-513.

Binswanger, H. P. (1981), Attitudes toward risk: Theoretical implications of an experiment in rural India, Economic Journal, 91 (364), décembre, 867890.

Binswanger, H. P. (1980), Attitudes toward risk: Experimental measurement in rural India, American Journal of Agricultural Economics, 62, 395407.

Binswanger, H. P. et Rosenzweig, M. R. (1986), Behavioral and material determinants of production relations in agriculture, Journal of Development Studies, 22, 503-539.

Binswanger, H. P. et Sillers, D. A. (1983), Risk aversion and credit constraints in farmers decision-making: A reinterpretation, Journal of Development Studies, 20 (1), octobre, 5-21.

Bonjean, C. (1994), Stabilisation des prix agricoles : quels bénéfices pour les producteurs? Études de cas dans M. Benoit-Cattin, M. Griffon et P. Guillaumont (éd.), Économie des politiques agricoles dans les PVD, t. 2, Paris, Éditions de la Revue française d'économie.

Bonjean, C. (1992), Instabilité des marchés agricoles et stratégies paysannes au Niger, Économie rurale, n" 210, juillet-août, 17-22.

Boussard, J.-M. (1996), When risk generate chaos, Journal of Economic Behavior and Organization, 29, 433-446.

Boussard, J.-M. (1995), Les droits de produire: justification économique, Compte rendu de l'Académie d'Agriculture de France, 81 (6), 67-78.

Boussard, J.-M. (1994), Revenus, marchés et anticipations : la dynamique de l'offre agricole, Économie rurale, n' 220-221, mars-juin, 61-68.

Boussard, J.-M. (1993), Dynamique des marchés et rôle de l'État en agriculture, Compte rendu des séances de l'Académie d'Agriculture de France, 79 (7), 53-68.

Boussard, J.-M. (1987), Économie de l'agriculture, Paris, Economica. 
Boussard, J.-M. et Gérard, F. (1996), Price stabilization and agricultural supply, in M. Benoit-Cattin, M. Griffon et P. Guillaumont (éd.), Economics of agricultural policies in developping countries, Paris, Éditions de la Revue française d'économie, 269-287.

Boussard, J.-M. et Petit, M. (1967), Representation of farmers behavior under uncertainty with a focus-loss constraint, Journal of Farm Economics, 49, 869-880.

Boussard, J.-M. et Piketty, M. G. (2000), Can market support trade ? The case of sugar, Communication au Congrès de l'IAAE, Berlin (à paraître) : A. Braverman, R. Kanbur, A. S. Brandao et M. Lopez (1990), Costs and benefits of agricultural price stabilization in Brazil, Working Papers, Agriculture and Rural Development Department, World Bank, décembre.

Chavas, J.-P. et Holt, M. T. (1993), Market instability and nonlinear dynamics, American Journal of Agricultural Economics, 75, 113-120.

Chtara, Yamina (1996), Aléas climatiques et fluctuations des prix des produits alimentaires en Afrique sahélienne; cas du Mali et du Niger pour les cultures de mil et de sorgho, Mémoire de DEA, Université de Paris I, Centre d'économie du développement, Paris.

Collier, P. et Gunning, J. W. (1997), Explaining african economic performance, mimeo, Centre for the Study of African Economies, University of Oxford.

Combes, J.-L. (1993), Instabilité des revenus et épargne dans les pays en voie de développement: le rôle de la politique de stabilisation du prix des produits agricoles d'exportation, Thèse de doctorat de l'Université de Clermont I.

Deaton, A. S. (1990), Saving in developing countries: Theory and review, World Bank Economic Review, Proceedings of the World bank Annual Conference on Development Economics, 61-96.

Deaton, A. S. (1991), Saving and liquidity constraints, Econometrica, 59, 1221-1248.

Deaton, A. S. (1992), Saving and income smoothing in Côte-d'Ivoire, Journal of African Economies, 1 (1),1-24.

Deaton, A. S. (1992), Commodity prices, stabilization and growth in Africa, Discussion Paper n* 166, research Program in Development Studies, Center of International Studies, Princeton University, décembre, 24.

Deaton, A. et Laroque, G. (1992), On the behaviour of commodities markets, Review of Economic Studies, 59, janvier, 1-23.

Dillon, J. L. et Scandizzo, P.-L. (1978), Risk attitudes of subsistence farmers in northeast Brazil : A sampling approach, American Journal of Agricultural Economics, août, 425-435.

Fafchamps, M. (1992), Cash crop production, food price volatility and rural market integration in the Third World, American Journal of Agricultural Economics, 74, 90-99.

Finkelshtain, I. et Chalfant, J. (1991), Marketed surplus under risk : Do peasants agree with Sandmo?, American Journal of Agricultural Economics, $73,557-567$. 
Friedman, M. (1954), The reduction of fluctuations in the income of primary producers : A critical comment, Economic Journal, 64, 698-703.

Guillaumont, P. (éd.) (1998), Évaluation globale du Stabex, CERDI.

Guillaumont, P. et Bonjean, C. (1991), Effects on agricultural supply of producer price level and stability with and without goods scarcity: The case of coffee supply in Madagascar, Journal of International Development, $3(2), 115-133$.

Guillaumont, P. et Combes, J.-L. (1994), Les effets de la tendance et de l'instabilité des prix payés aux producteurs sur la croissance de la production agricole d'exportation : analyse transversale, dans $\mathbf{M}$. Benoit-Cattin, M. Griffon et P. Guillaumont (éd.), Économie des politiques agricoles dans les PVD, t. 2, Paris, Éditions de la Revue française d'économie.

Guillaumont, P. et Guillaumont Jeanney, S. (1994), Ajustement et développement: l'expérience des pays ACP, Paris, Economica.

Guillaumont, P. et Guillaumont, S. (1990), Why and how to stabilize producer prices for export crops in developing countries, UNDP, World Bank Trade Expansion Program, Occasional Paper n" 6.

Gouin, D. M. et Morisset (1992), Le marché des quotas laitiers au Québec. La recherche de la concurrence parfaite, Économie rurale, 212 , 27-33.

Hardaker, J. B., Huime, R. B. M. et Anderson, J. R. (1997), Coping with risk in agriculture, $\mathrm{CAB}$ international, Wallingford (UK).

Hueth, D. L. et Furtan, W. H. (1994), The economics of agricultural crop insurance theory and evidence, Boston, Kluwer Academic.

Just, R. E. (1974), An investigation of the importance of risk in farmer decisions, American Journal of Agricultural Economics, février, 14-25.

Just, R. E. (1975), Risk aversion under profit maximization, American Journal of Agricultural Economics, 57, 347-352.

Kimball, M. S. (1990), Precautionary saving in the small and in the large, Econometrica, 58 (1), janvier, 53-73.

Laffont, J.-J. (1985), Cours de théorie micro-économique: économie de l'incertain et de l'information, Paris, Economica.

Lebosse, J.-C. et Ouisse, M. (1974), Les politiques d'intégration de l'agriculture artisanale au mode de production capitaliste, Économie rurale (102), $1-10$.

Le Bihan, J. (1960), L'intégration verticale: le point de vue des producteurs agricoles, Économie rurale (44), 100-120.

Lele, U. (1990), MADIA general report, Washington, World Bank.

Martineu, P. et Tissot, H. (1993), La répartition géographique des aléas climatiques, ENGREF, Paris (travail d'élève).

Massell, B. F. (1969), Price stabilization and welfare, Quaterly Journal of Economics, 38, 284-298.

Morduch, J. (1992), Risk, production and savings: Theory and evidence from indian households, Mimeo, Department of Economics, Harvard University. 
Mosacardi, E. et Janvry, A. de (1977), Attitudes toward risk among peasants : An econometric approach, American Journal of Agricultural Economics, $59,710-716$.

Munier, Bertrand R. (éd.) (1988), Risk decision and rationality, Reidel Dordrecht.

Newbery, D. (1989), The theory of food price stabilisation, Economic Journal, 99, décembre, 1065-1082.

Newbery, D. (1989), Agricultural institutions for insurance and stabilization, in P. Bardhan (éd.), The economic theory of agrarian institutions, Oxford, Clarendon Press.

Newbery, D. et Stiglitz, J. (1981), Theory of commodity price stabilization, Oxford, Clarendon Press.

Nubukpo, K. K. (1994), L'impact des aléas climatiques sur l'instabilité des marchés de céréales en Afrique de l'Ouest, Mémoire de DEA, GRID ENS Cachan.

Oi, W. Y. (1961), The desirability of price instability under perfect competition, Econometrica, 29 (1), 58-61.

Otsuka, K., Chuma, H. et Hayami, Y. (1992), Land and labor contracts in agrarian economies: Theorie and facts, Journal of Economic Litterature, 30, décembre, 1965-2018.

Paxson, C. (1992), Using weather variability to estimate the response of savings to transitory income in Thaïlande, American Economic Review, $82(1), 15-33$.

Ravallion, M. (1987), Markets and famines, Oxford, Clarendon Press.

Rosenzweig, M. R. et Binswanger, H. P. (1993), Wealth, weather risk and the composition and profitability of agricultural investments, Economic Journal, 103, janvier, 56-78.

Rotschild, M. et Stiglitz, J. E. (1970), Increasing risk I : A definition, Journal of Economic Theory, 2, 225-243.

Roumasset R., Boussard, J.-M., Singh J. (éd.), Risk and agricultural development, Agricultural Development Council, 1979.

Ruf, T. (1996), Boom et crises du cacao: les vertiges de l'or brun, Paris, L'Harmattan.

Sadoulet, E. et Janvry, A. de (1995), Quantitative Development Policy Analysis, Baltimore et Londres, Johns Hopkins University Press.

Udry, C. (1990), Credit markets in northern Nigeria : Credit as insurance in a rural economy, World Bank Economic Review, 4 (3), 251-269.

Waugh, F. V. (1944), Does the consumer benefit from price instability?, Quaterly Journal of Economics, 58 (3), 602-614.

Wolpin, K. I. (1982), A new test of the permanent income hypothesis: The impact of weather on the income and consumption of farm households in India, International Economic Review, 23, 583-594. 